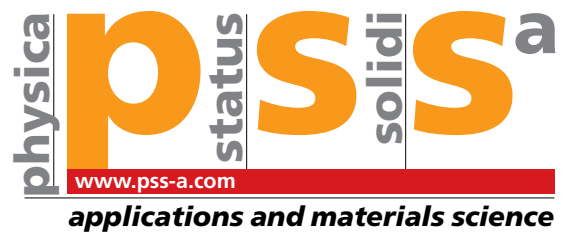

\title{
Immersion scatterometry for improved nano-scale topography measurements
}

\author{
Elson Liu and Fred L. Terry, Jr. \\ Department of EECS, University of Michigan, Ann Arbor, MI, USA
}

Received 10 June 2007, revised 5 January 2008, accepted 19 January 2008

Published online 26 March 2008

PACS 85.40.Hp, 87.64.Cc

*Corresponding author: e-mail fredty@umich.edu

Spectroscopic ellipsometry measurement of periodic structures ("scatterometry") has become a standard method for critical dimension and topography measurement in the integrated circuit industry. As dimensions are reduced, this method may not be adequate for research and process control.
Use of immersion mode measurements may extend the usefulness of near UV specular scatterometry. In this paper, we present the first experimental measurements of gratings in water immersion and discuss the issues in achieving improved nano-topography measurements with this method.
1 Introduction Specular-mode spectroscopic ellipsometry (SE) or reflectometry (SR) measurements from periodic gratings ("scatterometry", or optical critical dimension metrology-OCD) have been successfully demonstrated as accurate methods for extracting detailed topography of integrated circuit structures. This method is currently being used as an in-line metrology tool for waferto-wafer process monitoring and control in lithography and etch processes. However, as critical dimensions become very much less than the current minimum measurement wavelengths and as film thicknesses are simultaneously reduced, there are significant questions regarding the usefulness of this method for nanoelectronic device manufacturing control as critical dimensions approach $30 \mathrm{~nm}$ and below $[1,2]$. Reducing the wavelength of the measurement light below $190 \mathrm{~nm}$ into the VUV range not only poses major instrumentation challenges, but also presents problems with high energy photon damage of important sensitive films such as photoresists. Similar to the problems of electron beam damage during SEM measurements, the dimensions and optical properties of the photoresist lines can change during the course of measurement. One possible approach for improving the resolution of near UV-visible scatterometry is the use of higher index immersion fluids. This issue has been theoretically addressed in our earlier work [3]. Using a higher index immersion fluid yields two important advantages for scatterometry: (1) it reduces the effective measurement wavelength; and, perhaps more importantly, (2) allows real reflected modes for sub-100 nm period gratings. An interesting but more subtle effect is changing the coupling to transmitted diffracted modes. The major disadvantages include reduction of the optical contrast between the lines and the ambient, possible liquid absorption by the grating lines, possible trapped bubbles in the lines, and increased complexity. In this paper, we will present for the first time experimental results from both dry (air) and immersion (DI-H2O) measurements of photoresist gratings and will discuss the issues associated with obtaining higher resolution of critical dimensions and sidewall shapes.

2 Experimental All ellipsometry measurements were performed using a SOPRA GESP-5 rotating polarizer ellipsometer with a UV optimized prism/grating scanning monochromator. The measurements were conducted in tracking-analyzer mode and the data are reported in $\alpha, \beta$ format where $\alpha=\cos (2 \Psi)$ and $\beta=\sin (2 \Psi) \cos (4)$. Data were collected every $5 \mathrm{~nm}$ over the $225-820 \mathrm{~nm}$ range. Immersion measurements were performed using a cell machined from $\mathrm{Al}$ with fused silica windows. The windows were cemented in place using silicone rubber. Measurements were taken on a variety of uniform thin film and 
grating structures both on the standard sample stage and in immersion cell without fluid to verify that the windows had no significant effect on the collected data. The angle of incidence (AOI) for all measurements was $75.06^{\circ}$. The deviation from the nominal $75^{\circ}$ setting was determined by measurements over the $\lambda=500-820 \mathrm{~nm}$ range on a bare $\mathrm{Si}$ wafer (fitting the native oxide thickness and AOI).

Uniform $\mathrm{SiO}_{2}$ on $\mathrm{Si}$ samples were used as reference measurements. Two sets of photoresist (PR) grating samples were used for scatterometry. The first was a $700 \mathrm{~nm}$ period (nominally $350 \mathrm{~nm}$ line/space) PR grating on $31.7 \mathrm{~nm} \mathrm{SiO}_{2}$ on $\mathrm{Si}$ sample we had previously extensively characterized [4]. The other was a $160 \mathrm{~nm}$ period (nominally $80 \mathrm{~nm}$ line/space) grating on a bottom antireflective coating (BARC) layer supplied by Clariant AZ, Inc. We had previously measured the optical constants of the $700 \mathrm{~nm}$ resist on a similarly prepared uniform PR sample. The optical constants of the AZ resist and BARC layer were obtained by AZ from spectroscopic ellipsometry measurements. All grating measurements were done in the planar alignment configuration.

Immersion measurements were performed using high purity deionized water. The measurements were performed with the water stagnant, and no active temperature or ambient gas controls were used.

3 Results and discussion We measured the refractive index of $\mathrm{H}_{2} \mathrm{O}$ by measuring a thick $(\sim 2 \mu \mathrm{m}) \mathrm{SiO}_{2}$ on $\mathrm{Si}$ wafer both dry and wet. We simultaneously fit the oxide thickness and water index using the 4 parameter formulation of Tilton-Taylor [5]:

$$
n^{2}(\lambda)=a^{2}-k \lambda^{2}+\frac{m}{\lambda^{2}-l^{2}}
$$

In Ref. [5], the authors also parameterize the variation of these 4 parameters with temperature. To account for the possible affects of atmospheric gas absorption (principally $\mathrm{O}_{2}, \mathrm{~N}_{2}$ ), we used a Bruggemann effective media approximation (BEMA) mixture of Tilton-Taylor water at $23{ }^{\circ} \mathrm{C}$

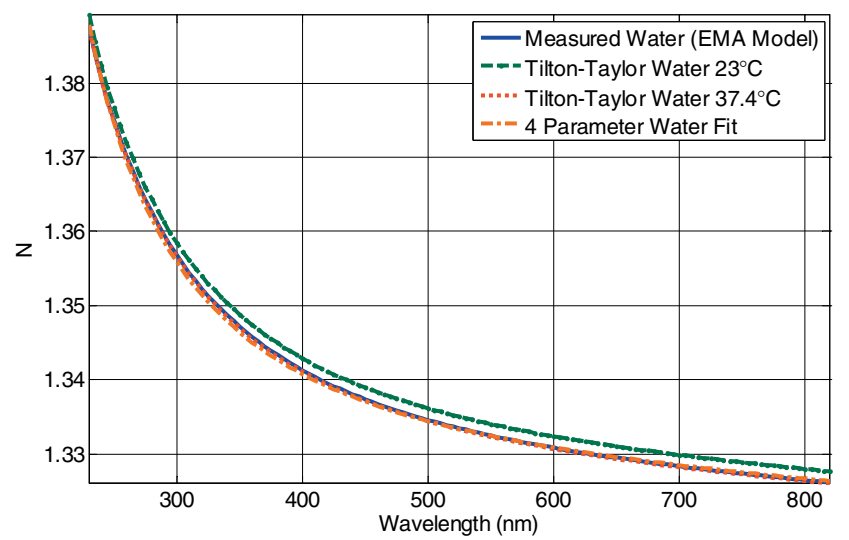

Figure 1 (online colour at: www.pss-a.com) Extracted water refractive index (solid blue curve) and Tilton-Taylor reference data at $23{ }^{\circ} \mathrm{C}$ (dashed green curve). and voids. We also investigated allowing all 4 of the Tilton-Taylor parameters to freely vary during regression. The result of one of these fits is shown in Fig. 1. The unbiased estimator of error, $\sigma$, for all these fit was $<0.03$. All 3 parameterizations yielded nearly identical results for our water. In particular, the BEMA fit with $0.46 \%$ voids perfectly overlays the Tilton-Taylor $37.4{ }^{\circ} \mathrm{C}$ fit. Since it was not physically possible for our laboratory and the immersion cell to be this hot, we would rule out temperature variation as the major cause of our water index variations. For the immersion fits for gratings, we used the BEMA model for all results presented in this paper. We also investigated varying all 4 Tilton-Taylor parameters in the grating cases, but this led to model over-parameterization and possibly nonphysical results for the water index. The extracted void fractions extracted in our fits were below those suggested by $\mathrm{O}_{2}$ and $\mathrm{N}_{2}$ solubilities in $\mathrm{H}_{2} \mathrm{O}$ [8]. Changes in the water refactive index due to contamination cannot be ruled-out, but this seems unlikely based on the results of studies of aqueous ion solutions [9]. Further, we see apparent reductions in the water refractive index, whereas ionic contamination would tend to raise it.

The $700 \mathrm{~nm}$ PR grating samples were first characterized using measurements in air. The line topography was extracted using Levenberg-Marquardt-based regression analysis. The grating model used the Rigorous Coupled Wave Analysis (RCWA) algorthm [6] with continuity corrections to improve the p-polarization (TM mode) convergence [7]. For the $700 \mathrm{~nm}$ gratings, the line was divided into equal height trapezoids. First a single trapezoid was fit to the data. This best-fit result was used to seed a 2 trapezoid fit, then a 4 trapezoid fit, etc. We stopped at an 8 trapezoid fit (further division would have led to overfitting). This yielded a 10 parameter (height +9 widths) fit as illustrated in Fig. 3 with results summarized in Table 1.
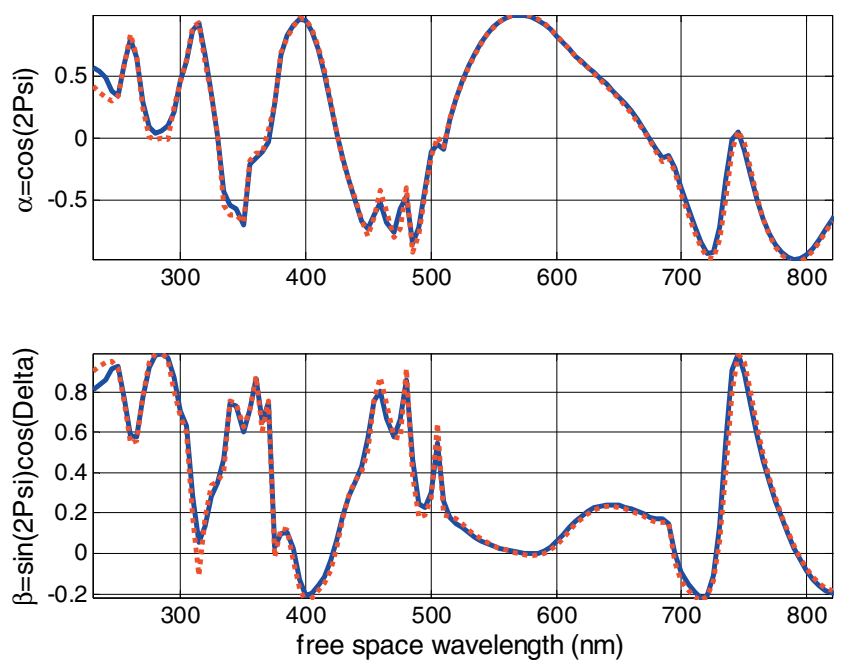

Figure 2 (online colour at: www.pss-a.com) Measured (blue solid curves) and fitted (dashed green curves) SE data for $700 \mathrm{~nm}$ PR grating measured in air. The unbiased estimator of error for this fit, $\sigma$, was $\sim 0.0478$. 


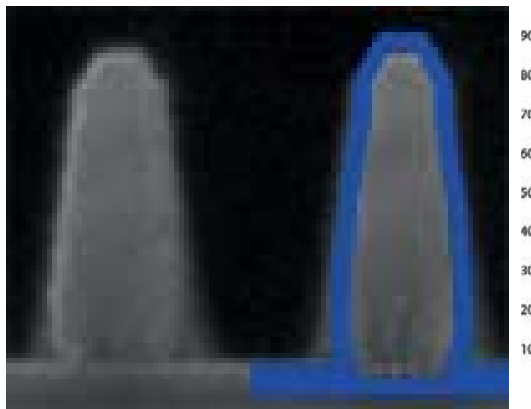

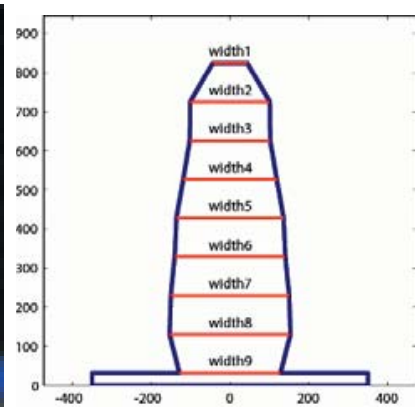

Figure 3 (online colour at: www.pss-a.com) Scanning electron microscopy (SEM) cross-section and scatterometry fit overlay for data of Fig. 2.
For these fits, 11 spatial orders were used in the RCWA simulation (based on convergence tests) and a slicing algorithm that insured no more than a $2 \mathrm{~nm}$ width change between each slice (resulting in 159 slices for the fit in Figs. 2 and 3 ).

The sharp structure in this data arises from scattering of energy into non-specular reflected and transmitted modes. For the $700 \mathrm{~nm}$ gratings measured in air, simulations from the fitted structures show significant scattering into the $m=1-6$ backscattered modes (both reflected and transmitted). Forward scattering is insignificant for these structures over the wavelength measured. Strong transmitted mode scattering tends to occur just before the allowed onset of the same order reflected mode (as illustrated in Fig. 4). This trend occurred for all PR grating structures studied. These regions of strong diffraction are very sensitive to changes in the grating lineshape.

In immersion mode, the backscattered $m=1-8$ modes are allowed and the areas of sharp structure for each mode are shifted to a lower wavelength as governed by the grating equation:

$$
\sin \theta_{\mathrm{D}}=\sin \theta_{\mathrm{i}}+\frac{m \lambda_{0}}{\Lambda n_{\mathrm{I}}}
$$

where $\theta_{\mathrm{D}}$ is the angle of diffraction, $\theta_{\mathrm{i}}$ is the angle of incidence, $m$ is mode order, $\lambda_{0}$ is the free space wavelength, $\Lambda$

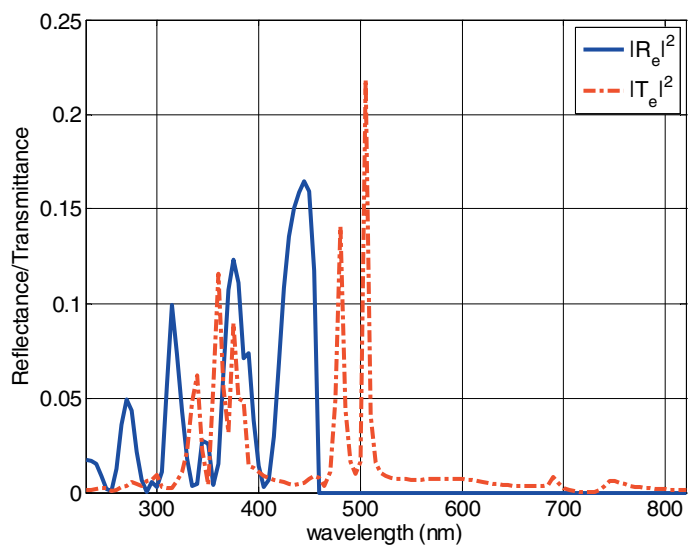

Figure 4 (online colour at: www.pss-a.com) Simulated $m=3$ backscattered reflected (blue-solid) and transmitted energy (reddashed) for fit shown in Figs. 2 and 3. is the grating period, and $n_{\mathrm{I}}$ is the ambient index of refraction. Measurement of the same $700 \mathrm{~nm}$ grating in water yields the measured data and fit in Figs. 5 and 6. Since the dry data topography fit agrees well with SEM observations, we must conclude that there is a systematic error in either the immersion measurement or the ambient refractive index extraction. Our simulations indicate that an angle of incidence error will not explain this variation. Also, measurement of different thicknesses of $\mathrm{SiO}_{2}$ on Si show very good quality fits in both dry and immersion measurements with the same oxide thickness. Variations in the photoresist density were investigated (assuming an effective media mixing of voids with the reference PR index data), but this too failed to explain our observed immersion measurements. Also, including trapped air (bubbles) in the grating lines also failed to prove valid.

To best fit our current experimental data, we simultaneously fit a single topography to both the dry and immersion data and also varied the water index using the BEMA model. The topography extracted from this fit very close overlay that of the dry only fit as shown in Fig. 7. The extracted void fraction of the water was $0.537 \pm 0.087 \%$. The values of the extracted topography parameters are very close to those of Table 1 . The confidence limits are slightly higher. The additional information in the immersion measurement did not offset the additional uncertainty introduced by adding an additional model parameter.

Table 1 Fit results and confidence limits for topography fit shown in Figs. 2 and 3.

\begin{tabular}{lrc}
\hline parameter & fit (nm) & $95.4 \%$ confidence \\
\hline height & 793.04 & 2.39 \\
width 1 (top) & 86.97 & 8.84 \\
width 2 & 201.95 & 10.09 \\
width 3 & 203.93 & 5.43 \\
width 4 & 237.00 & 5.93 \\
width 5 & 271.89 & 7.06 \\
width 6 & 280.65 & 9.19 \\
width 7 & 300.36 & 10.81 \\
width 8 & 306.67 & 10.66 \\
width 9 & 256.97 & 14.44 \\
\hline
\end{tabular}



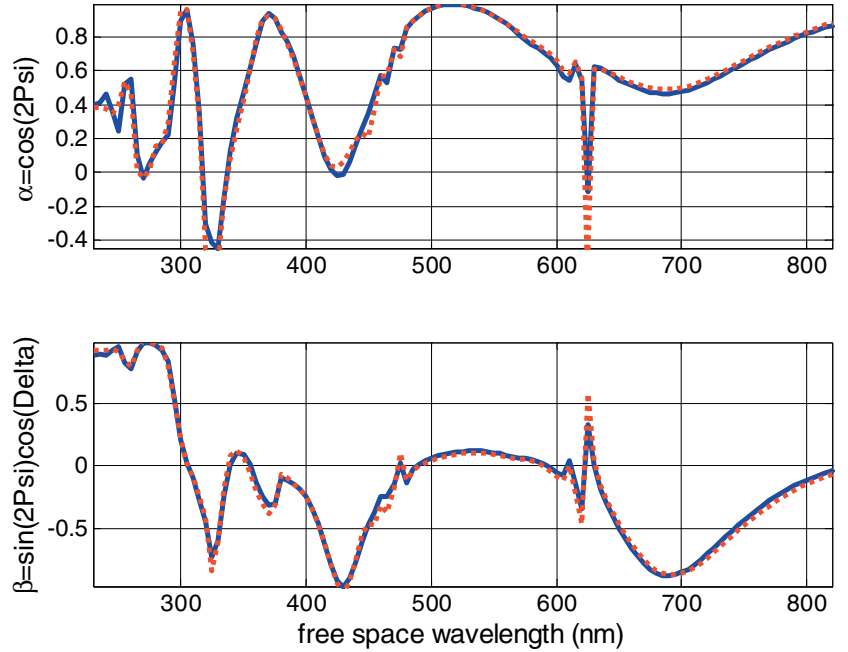

Figure 5 (online colour at: www.pss-a.com) $\mathrm{H}_{2} \mathrm{O}$ Immersion mode measurement (solid) and regression fit (dotted) to the same $700 \mathrm{~nm}$ PR grating sample as Fig. $2 \sigma \sim 0.053$.

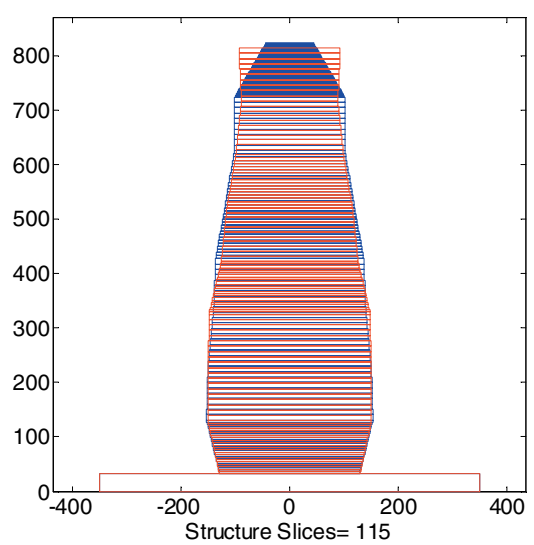

Figure 6 (online colour at: www.pss-a.com) Resulting fit (red) from the immersion data of Fig. 4 overlaid on the fit from the dry fit (blue) of Fig. 3.

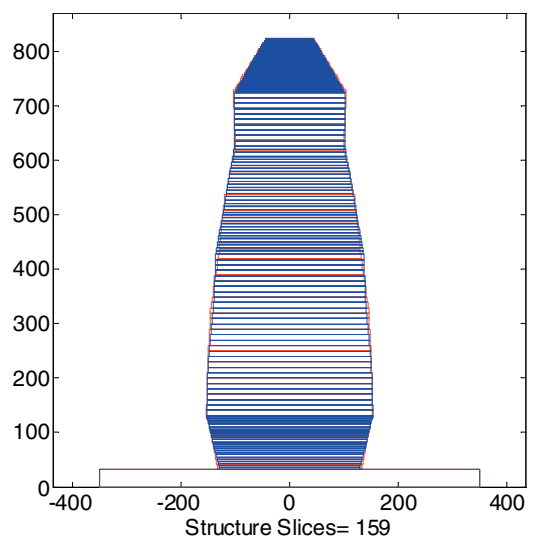

Figure 7 (online colour at: www.pss-a.com) Dry only fit (blue) and dual ambient/BEMA fit (red) for the $700 \mathrm{~nm}$ grating.
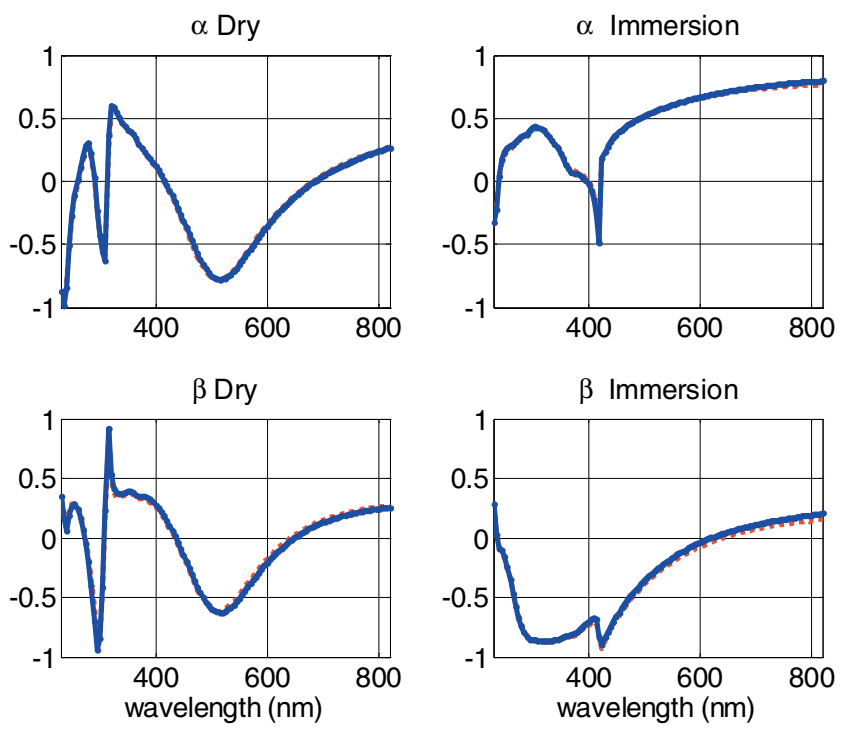

Figure 8 (online colour at: www.pss-a.com) Dry (lef) and immersion (right) $\mathrm{SE}$ data from the $160 \mathrm{~nm}$ period gratings. The data (blue-solid) is shown along with the single trapezoid fit (reddashed). The onset of the first order backscattered mode can be clearly seen in the sharp structure of both the wet and dry measurements.

For the $160 \mathrm{~nm}$ PR gratings, only the $1^{\text {st }}$ order backscattered mode is accessible $(\lambda<315 \mathrm{~nm}$ dry, $430 \mathrm{~nm}$ in $\mathrm{H}_{2} \mathrm{O}$ ). This lack of allowed scattered modes reduces the sensitivity to the lineshape. The measured data could be fit well using a single trapezoid model as shown in Fig. 8 and with the results shown in Table 2. The dual ambient fit is very similar to a dry-only fit for the single trapezoid shape model. Our previous computational studies showed that additional shape information should be obtainable from the extra information in immersion data [3].

The problems posed by the apparent variation of water refractive indices prevented us from experimentally demonstrating improved confidence limits in the extracted topography parameters. However, in our attempts to gain more shape information from the $160 \mathrm{~nm}$ period gratings, we did find a strong indication that the extra information in the immersion data may be helpful. We fit the data using increasing numbers of equal height trapezoids. As the number of trapezoids in the model increased, we found that the dry fits would deviate into physically unreasonable structures while the dual mode (dry/wet-BEMA) fits

Table 2 Fit results and confidence limits for topography fit shown in Fig. 8. $\sigma=2.5 \times 10^{-2}$ for this fit.

\begin{tabular}{lrl}
\hline parameter & \multicolumn{1}{l}{ fit } & $95.4 \%$ confidence \\
\hline height $(\mathrm{nm})$ & 138.57 & 0.50 \\
top width $(\mathrm{nm})$ & 49.38 & 0.85 \\
bottom width (nm) & 55.04 & 1.76 \\
BARC thickness (nm) & 39.53 & 0.18 \\
void fraction in water (\%) & 1.01 & 0.30 \\
\hline
\end{tabular}




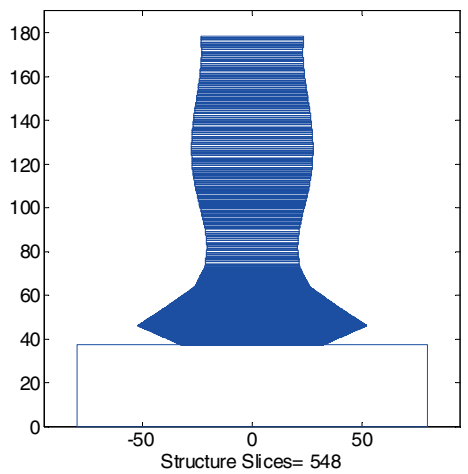

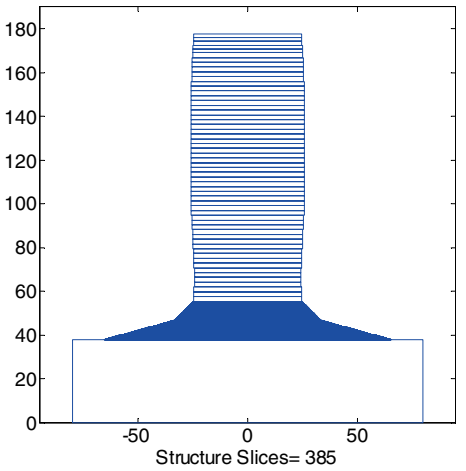

Figure 9 (online colour at: www.pssa.com) 16 trapezoid fits to the $160 \mathrm{~nm}$ period gratings for dry-only data (left) and dual (dry/wet-BEMA) (right). yielded physically reasonable resist shapes. An illustration of this is found in Fig. 9 for a 16 trapezoid fit. Clearly, this is an over-parameterization and the results may be nonphysical in both cases; however, it is interesting to note that the dry-only fit is very improbable for a photoresist structure, whereas the dual mode fit yields the commonly encountered case of a basically vertical line with a "footer." Due to the very small size of these features, we have not yet been able to obtain cross-sectional electron micrographs to further investigate this result.

4 Conclusions In this paper, the first immersion mode measurements and topography extraction from grating structures were presented. The measured results confirm the basic validity of our earlier computational modelling [3]. Experimental errors prevented us from clearly demonstrating enhanced resolution results in this work, but the $160 \mathrm{~nm}$ grating results do indicate that the extra scattering information in the immersion data is beneficial.

It is not surprising that no benefit was observed for the $700 \mathrm{~nm}$ period gratings. Since a relatively large number of scattered modes are accessible in air, the relative shape sensitivity of the dry measurement is already very high. For the $160 \mathrm{~nm}$ gratings, the added scattering data from the immersion measurement significantly adds to the shape sensitivity. Reducing the systematic errors in the immersion measurement should lead to significant further im- provement. In particular, we will employ standard methods for degassing our deionized water and will use a filled, fully enclosed cell to minimize gas absorption during measurements.

Acknowledgements Support of this work by the KLATencor Corporation is gratefully acknowledged. We thank J. Bendik for valuable discussions and aid with samples. We also thank A. Romano of Clariant AZ for the $160 \mathrm{~nm}$ samples and materials data.

\section{References}

[1] F. L. Terry, Jr., Proc. SPIE 5038, 547 (2003).

[2] B. J. Rice, H. Cao, O. Chaudhuri, M. Grumski, B. Hartenek, A. Liddle, D. Olynick, and J. Roberts, Proc. SPIE 5375, 183 (2004).

[3] F. L. Terry, Jr. and J. J. Bendik, Proc. SPIE 5752, 237 (2005).

[4] H.-T. Huang and F. L. Terry, Jr., Thin Solid Films 455/456, 828 (2004).

[5] L. W. Tilton and J. K. Taylor, J. Res. Natl. Bur. Stand. 20, 419 (1938).

[6] M. G. Moharam, D. A. Pommet, E. B. Grann, and T. K. Gaylord, J. Opt. Soc. Am. A 12, 1077 (1995).

[7] L. Li, J. Opt. Soc. Am. A 13, 1870 (1996).

[8] C. J. J. Fox, Trans. Faraday Soc. 5, 68 (1909).

[9] T. Berlind, G. K. Pribil, D. W. Thompson, J. A. Woollam, and H. Arwin, phys. stat. sol. (c) 5, 1249 (2008), these proceedings. 